\title{
Enhanced quality and transparency of health research reporting can lead to improvements in public health policy decision-making: help from the EQUATOR Network
}

Evidence published in the scientific literature is commonly used in public health to support policy decisions. A wide variety of stakeholders consults Cadernos de Saúde Pública (CSP) to assess and summarize research evidence, plan health programs, design new interventions, and identify risk factors for a particular health outcome. Thus, it is crucial that scientific articles are published transparently and accurately in order to allow evaluating the reliability of the research for future use.

CSP receives more than 1,500 research manuscripts annually, and a high proportion fail to achieve acceptable quality levels for publication, either because the study was poorly conducted or reported, or both. Common flaws include lack of control of confounding in observational studies, lack of information on quality assessment for studies included in systematic reviews, and poor descriptions of clinical trial interventions. Biases due to deficiencies in the performance and reporting of health research can be dangerously misleading to policy decisions. The literature provides abundant examples, such as the erroneous belief that hormone replacement therapy protects against cardiovascular disease, a statistical relationship that was ultimately proven to be confounded by socioeconomic status (von Elm EV, Egger M. BMJ 2004; 329:868-9).

CSP publishes numerous observational studies, systematic reviews, clinical trials, among others. Unfortunately, only a small portion of submitted manuscripts use appropriate reporting guidelines such as Strengthening the Reporting of Observational studies in Epidemiology (STROBE), Preferred Reporting Items for Systematic Reviews and Meta-Analyses (PRISMA), or Consolidated Standards of Reporting Trials (CONSORT). Compliance with these guidelines is a great way to improve transparency and accuracy and has proven to increase the reliability and impact of health research (Simera et al. BMC Med 2010; 8:24).

The EQUATOR Network (Enhancing the QUAlity and Transparency Of health Research) is "an international initiative that seeks to improve the quality of scientific publications". EQUATOR (http://www.equator-network.org/) has compiled a vast catalogue of all available reporting guidelines in its library to help authors and peer reviewers of health research. It also provides education and training.

EQUATOR works with a variety of key stakeholders in the research and publication process including journal editors, peer reviewers and developers of reporting guidelines. The Pan-American Health Organization has recently established a formal agreement with EQUATOR to disseminate the initiative in Latin America. It is already possible to browse a Spanish version of the Network's website. Several reporting guidelines have also been translated into Spanish.

In the near future, CSP may only publish articles that follow appropriate reporting guidelines. It is time to reduce wastefulness and fully realize the investment in health research by translating findings from high quality research into health benefits derived from evidenced-based policy decisions.

Gilberto Kac

Instituto de Nutrição, Universidade Federal do Rio de Janeiro,

Rio de Janeiro, Brasil.

kacetal@gmail.com
Allison Hirst

EQUATOR Network, University of Oxford, Oxford, UK. 